\title{
Patient and Public Preferences for Treatment Attributes in Parkinson's Disease
}

\author{
Marieke G. M. Weernink ${ }^{1} \cdot$ Janine A. van Til $^{1} \cdot$ Catharina G. M. Groothuis-Oudshoorn $^{1}$ \\ Maarten J. IJzerman ${ }^{1}$
}

Published online: 15 May 2017

(C) The Author(s) 2017. This article is an open access publication

\begin{abstract}
Background Patient and public preferences for therapeutic outcomes or medical technologies are often elicited, and discordance between the two is frequently reported.

Objective Our main objective was to compare patient and public preferences for treatment attributes in Parkinson's disease (PD).

Methods A representative sample from Dutch PD patients and the general public were invited to complete a best-worst scaling case 2 experiment consisting of six health-related outcomes and one attribute describing the specific treatment (brain surgery, pump, oral medication). Data were analyzed using mixed logit models, and attribute impact was estimated and compared between populations (and population subgroups).

Results Both the public $(N=276)$ and patient $(N=198)$ populations considered treatment modality the most important attribute, although patients assigned higher relative importance. Both groups assigned high disutility to pump infusion and brain surgery and preferred drug treatment. Most health outcomes were valued equally by patients and the public, with the exception of reducing dizziness (more important to the public) and improving slow movement (more important to patients).
\end{abstract}

Electronic supplementary material The online version of this article (doi:10.1007/s40271-017-0247-7) contains supplementary material, which is available to authorized users.

Marieke G. M. Weernink

m.g.m.weernink@utwente.nl

1 Department of Health Technology and Services Research, MIRA Institute for Biomedical Technology and Technical Medicine, University of Twente, P.O. Box 217, 7500 AE Enschede, The Netherlands
Discussion Although these data do not support definite conclusions on whether patients are less likely to undergo invasive treatments, the (predicted) choice probability of undergoing brain surgery or having pump infusion technology would be low based on the (un)desirability of the attribute levels. Patients with PD might have adapted to their condition and are not willing to undergo advanced treatments in order to receive health improvements. Both public and patient preferences entail information that is potentially relevant for decision makers, and patient preferences can inform decision makers about the likelihood of adaptation to a specific condition.

\section{Key Points}

Both the public and patient populations consider treatment modality the most important attribute, although patients assigned higher relative importance.

Most health outcomes were valued as equally important by patients and the public, with the exception of dizziness (higher public value) and slow movement (higher patient value).

Both public and patient preferences entail information that is potentially relevant for decision makers, and patient preferences can inform decision makers about the likelihood of adaptation to a specific condition.

\section{Introduction}

Parkinson's disease (PD) is a chronic neurodegenerative disease for which no curative treatment is available. Many symptoms and complications accompany PD, such as slower movement (bradykinesia), resting tremor, muscular 
rigidity, and dyskinesia, and symptomatic treatment aims to improve the patient's quality of life and functional capacity [1-3]. Early-stage PD is predominantly treated with drugs; however, recent developments to extend therapeutic options for patients with more advanced PD include novel approaches such as continuous pump infusion technology (intraduodenal or subcutaneous) and brain surgery techniques (deep brain stimulation) [4]. The device-aided therapies are effective in reducing motor symptoms, but currently no direct comparative data exist to support the use of one therapy over another [5].

In addition, new technologies are systematically evaluated on their social, economic, organizational and ethical consequences in healthcare [6-8]. While such health technology assessment is performed from a public perspective on the need for reimbursement of these technologies [7, 8], it is sometimes argued that patients' preferences should be considered. Patients have experience with the condition and can provide unique knowledge that can support more informed decision making on the need for reimbursement [9].

Although discordance is frequently reported between patient and public preferences, no preference studies are available that compare patient and public valuations for clinical outcomes of PD technologies [10, 11]. Therefore, the first objective of this study was to compare patient and public preferences for clinical outcomes (symptom reduction, side effects) of PD treatment. It is hypothesized that patients will place higher importance on the burden of treatment because they have experience with side effects (in contrast to the public).

Besides maximization of the treatment's effect on health outcomes, evaluating the process of care can also be of paramount importance $[12,13]$. Especially in evaluations in chronic diseases, the possibility exists that discomfort of treatment can outweigh treatment benefits. Hence, besides the effectiveness of a drug, other attributes such as ease of use and treatment modality influence its use by patients and, through adherence, its efficacy [14, 15]. For the newer technologies in PD, it is particularly interesting to study the relative trade-offs between the process of care (taking pharmaceuticals, using a pump infusion, or undergoing brain surgery) and improved health outcomes. Therefore, the second objective of this study was to compare the impact of treatment modality on treatment desirability from both the patient and public perspective. It is hypothesized that patients have a more positive view of pump infusion and brain surgery because they might be more willing to adapt in order to receive improved symptom reduction.

Lastly, preferences have the tendency to be influenced by sociodemographic factors and experience [16-18]. In an accompanying study, we identified different preference patterns for patients with PD who had experience with advanced treatments compared with patients who had no experience [19]. In general, public preferences are regarded as ex-ante preferences that are obtained without any experience [20]. However, individuals may have more or less experience with PD depending on their social environment, or may find it easier to relate to PD patients depending on their age or declining health. Hence, the last objective was to explore whether public preferences differ based on sociodemographic variables or the public respondents' familiarity with the disease.

\section{Methods}

\subsection{Selection of Attributes and Levels}

The first step in each preference study is to select attributes and levels. The identification of relevant clinical outcomes took place through qualitative interviews with 18 patients. Patients were asked which symptoms, side effects, or actions were mostly impeding their daily life (e.g. difficulty walking, dizziness, and taking pills). The interviews provided the basis for identifying the full set of attributes that characterize PD treatments and influence the patients' health-related quality of life (HRQoL). A project team consisting of a pharmacist, neurologist, rehabilitation specialist, two patients, and researchers discussed the interview results and selected less than eight attributes because the human mind is limited in its ability to include many attributes and levels in decision making. The selected attributes had to be a balanced set of symptoms, side effects, and process characteristics based on the qualitative and quantitative importance of these treatment attributes in the patients' daily life (interview results). Furthermore, the project team had to make sure that the selected attributes were typical symptoms and side effects of PD and not too general (e.g. nausea). They eventually selected six clinical outcomes: three symptoms (tremor, slowness of movement, posture and balance problems) and three side effects (dizziness, drowsiness, and dyskinesia). To minimize the cognitive difficulty for respondents, and to frame the treatment profiles as comprehensively as possible (especially important for the patients), the choice was made to have a similar and comprehensive level designation for clinical outcomes: 'seldom', 'sometimes', 'often suffering from'. Furthermore, one process attribute was added to refer to the treatment modalities of the main treatment options (oral medication, continuous infusion of medication via a pump, and undergoing neurosurgery). Table 1 displays the selected attributes and levels (a more detailed description of this preliminary research can be found in "ESM Appendix A"). 


\subsection{Design of the Preference Instrument}

Multiple techniques are available to measure the relative importance of treatment aspects in PD. More recently, best-worst scaling (BWS) was introduced as a way of obtaining more information by asking respondents to select not only the best alternative (most preferred) but also the least preferred alternative [21]. This best-worst question is repeated several times in certain combinations of attributes and levels to determine the relative importance of each item. Different types of BWS exist. BWS case 1 was not a relevant type of choice here as the attributes were not equivalent to item statements, but were in need of attribute levels to indicate the effect of treatment or the occurrence of side effects (e.g. seldom, sometimes, or often). BWS case 2 (the profile case) lets respondents evaluate one treatment scenario in one choice task and asks them to identify their most and least preferred attribute level. BWS case 3 is comparable to a discrete choice experiment (DCE) choice set and asks respondents to choose the best and the worst from a number of scenarios (usually three). Five pilot tests were conducted in our vulnerable patient group to compare the feasibility of BWS cases 2 and 3. Patients reported it was difficult to compare multiple treatment profiles in one choice task and suffered from information overload (BWS case 3). Subsequently, the project team had a preference for BWS case 2 (which was well-received by patients in the pilot tests). The next step was to select the hypothetical treatment scenarios. It was impossible to let respondents evaluate all possible 2187 scenarios $\left(3^{7}\right)$; therefore, Sawtooth Software (Sawtooth Software Inc., Orem, UT, USA) was used to select the smallest subset of treatment scenarios that was able to identify all necessary parameters. A D-efficient design of 36 unique treatment scenarios was selected, meaning that the design was balanced (each attribute level appears 12 times), but not completely orthogonal (meaning there is slight correlation between attribute levels). The 36 scenarios were then divided into four blocks (versions) of nine scenarios per respondent.

For each treatment scenario, respondents were asked to select the two aspects that they perceived as most and least desirable (Fig. 1). Treatment scenarios were formulated so that patients could recognize themselves in the description, and the public can evaluate what life would be like living while undergoing this treatment. The public sample was also specifically asked to imagine a situation in which they had been diagnosed with PD. Before answering these questions, both patients and public respondents received an extensive explanation and illustration of the selected

Table 1 Selected attributes and levels (descriptions)

\begin{tabular}{|c|c|c|}
\hline Attributes & Description & Level operationalization \\
\hline Treatment modality & The path by which a treatment enters (or is applied to) the body & $\begin{array}{l}\text { Oral medication } \\
\text { Continuous infusion of medication via a pump } \\
\text { Brain surgery }\end{array}$ \\
\hline Tremor & Rhythmic muscle contraction & $\begin{array}{l}\text { Seldom to never suffer from } \\
\text { Sometimes suffer from } \\
\text { Often suffer from }\end{array}$ \\
\hline $\begin{array}{l}\text { Posture and balance } \\
\text { problems }\end{array}$ & Abnormal axial postures and loss of postural reflexes & $\begin{array}{l}\text { Seldom to never suffer from } \\
\text { Sometimes suffer from } \\
\text { Often suffer from }\end{array}$ \\
\hline Slowness of movement & Difficulties with planning, initiating and executing movement & $\begin{array}{l}\text { Seldom to never suffer from } \\
\text { Sometimes suffer from } \\
\text { Often suffer from }\end{array}$ \\
\hline Drowsiness & Excessive daytime sleepiness & $\begin{array}{l}\text { Seldom to never suffer from } \\
\text { Sometimes suffer from } \\
\text { Often suffer from }\end{array}$ \\
\hline Dizziness & Lightheadedness, effect of orthostatic hypotension & $\begin{array}{l}\text { Seldom to never suffer from } \\
\text { Sometimes suffer from } \\
\text { Often suffer from }\end{array}$ \\
\hline Dyskinesia & Repetitive, involuntary muscle movement & $\begin{array}{l}\text { Seldom to never suffer from } \\
\text { Sometimes suffer from } \\
\text { Often suffer from }\end{array}$ \\
\hline
\end{tabular}


Fig. 1 Best-worst scaling exercise. $P D$ Parkinson's disease

\begin{abstract}
We would like to know your opinion on the desirability of the benefits and harms of possible treatments for Parkinson's disease.

Please look at the treatment description below. This is a description of a possible treatment for PD. How do you feel about this treatment?

For each treatment, we would like you to indicate the most desirable characteristic of treatment and the least desirable characteristic of treatment by ticking the respective boxes.
\end{abstract}

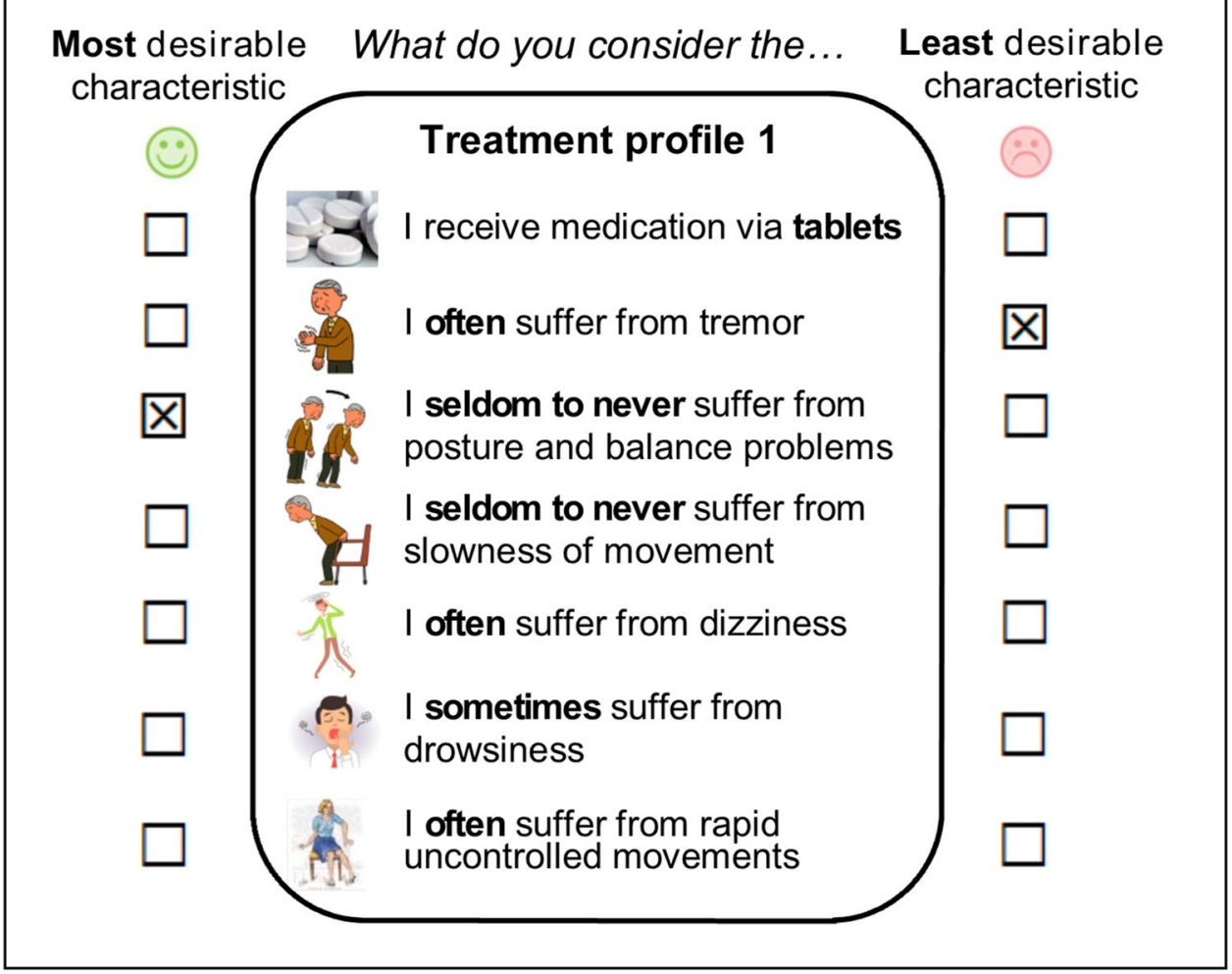

attributes, levels, and treatment for PD. They received a standard sheet with clinical background information about PD and factual information about the main treatment options (pharmaceutical treatment, neurosurgery, and receiving medication via a pump). Lastly, respondents were asked to answer sociodemographic questions regarding their sex, age, and education, and fill out the EuroQol-5D-5L health-related quality-of-life instrument [22]. The survey instrument can be found in "ESM Appendix B".

\subsection{Study Sample}

PD patients who were diagnosed with idiopathic PD at the outpatient department of the Medisch Spectrum Twente hospital in The Netherlands $(N=287)$ received a paper version of the BWS questionnaire at home. Patients who were registered as having atypical Parkinsonism or dementia did not receive an invitation. The data collection was expanded by recruiting respondents through online Parkinson communities, the website and paper magazine of the Dutch Parkinson's Disease Society, and PD forums. It could not be verified whether these participants were genuinely diagnosed with idiopathic PD or had atypical Parkinsonism or dementia because there was no access to hospital records. These patients were invited to complete the online survey or to request the paper version.

A Dutch public sample was recruited via an online survey sample coordinated by Survey Sampling International (Rotterdam, The Netherlands). Respondents, aged 18-65 years, were invited via e-mail to respond to the webbased questionnaire.

No literature is available to determine sample sizes for BWS experiments. Therefore, sample sizes were 
determined based on a rule of thumb for conjoint analysis that stated that estimate precision increases quickly at sample sizes over 150 and flattens out at \pm 300 observations [23, 24]. Taking into consideration the number of patients who received an invitation via the Medisch Spectrum Twente, we aimed to have at least 150 complete responses for both study samples. According to the Medical Ethics Committee of the Medisch Spectrum Twente, our study did not require assessment according to the Medical Research (Human Subjects) Act.

\subsection{Data Analysis}

Public respondents who did not complete the survey, or completed it within $5 \mathrm{~min}$, were excluded from analysis. Patient respondents were excluded if their paper questionnaire contained too many blank answers or response errors in the BWS choice tasks (ticked multiple best/worst). Differences in sociodemographic variables between the patient and general populations were identified with independent sample $t$ tests and Chi-square tests.

For the BWS analysis, the maximum difference (maxdiff) model was adopted in which it was assumed that respondents pick both best and worst items at the same time. Part-worth utilities for each attribute-level were obtained using a mixed (random parameter) logit model for each sample to account for the panel nature of the data [25]. Dummy coding was applied to each attribute. The model estimate for each attribute level was interpreted as a relative utility (relative to the reference level). The $p$ value indicates whether the attribute level significantly $(p<0.05)$ differs from the selected reference level.

The estimated utility weights for the patient and public populations cannot be directly compared because of the confound between mean and variance in the latent scale. Importance weights were therefore calculated, based on the difference between minimum and maximum part-worth utilities within an attribute. The largest difference value received an importance weight of one, representing the attribute that had the highest impact on treatment desirability; the other difference values were divided by the largest difference value, resulting in a relative distance of all attributes to the attribute with the highest impact [26]. In addition, to present a graphical insight in the value differences, we rescaled the part-worth utilities on a $0-1$ scale and presented a scatterplot. The attribute level with the lowest part-worth utility was anchored at 0 , and the attribute level with the highest part-worth utility was anchored at 1 (Eq. 1). Correlation between patient and public preferences was assessed using Pearson's correlation coefficient.
Rescaled value $\left(A_{i}\right)=\frac{U\left(A_{i}\right)-U\left(A_{\min }\right)}{U\left(A_{\max }\right)-U\left(A_{\min }\right)}$

where $U\left(A_{i}\right)$ represents the part-worth utility of an attribute level, $U\left(A_{\min }\right)$ is the attribute level with the lowest partworth utility, and $U\left(A_{\max }\right)$ is the attribute level with the highest part-worth utility.

Furthermore, explorative subgroup analyses were performed to examine whether public respondents were shaping and (re)constructing their preferences on the basis of their experiences (with PD). The variables of age, quality of life (EQ5D scores), and familiarity with PD were tested. Familiarity with PD was operationalized as being close to someone (i.e. family, friends) who suffers from PD. All data were analyzed using Stata version 14 (StataCorp LLC, College Station, TX, USA).

Please note that this article focuses on the differences between patient and public preferences, whereas the extensive clinical implications of patient preferences have been published elsewhere [19]. In addition, the public dataset has been used in an article to compare the outcomes of different preference methods in measuring process-related utilities [27].

\section{Results}

In total, 228 patients returned the paper questionnaire or completed the online survey. Some paper questionnaires were incomplete or contained multiple response errors and were excluded from analysis $(N=30,13.2 \%)$. Overall, 285 complete questionnaires were filled out online by the general public respondents (dropout rate $35 \%$ ), but nine respondents $(3.1 \%)$ were excluded because of the time criterion. The differences between the patient and public populations in the distribution of age, sex, education, and EQ5D-5L index value are displayed in Table 2.

\subsection{Preference for the Attribute Treatment Modality}

Table 3 shows the results of the BWS analysis: the partworth utilities could be interpreted relative to the selected reference level, often suffering from dyskinesia. The results show that treatment modality had the greatest impact on the perceived desirability of treatment in both the public and patient population (importance weight of 1) (Table 3); however, the patients assigned higher importance to treatment modality in comparison to the public population (demonstrated by the lower attribute impacts for the other attributes). We did not have prior expectations with regard to the relative ordering of treatment modalities, yet the scatterplot with the rescaled BWS data (Fig. 2) 
Table 2 Demographic details of study participants

\begin{tabular}{lccc}
\hline Variable & Patients $(n=198)$ & Public population $(n=276)$ & $p$ value \\
\hline Male sex & $133(67.2)$ & $98(35.5)$ & $<0.0001$ \\
Education $^{\mathrm{a}}$ & & & \\
Low & $70(35.4)$ & $52(18.9)$ & $<0.0001$ \\
Middle & $55(27.8)$ & $119(43.3)$ & \\
High & $73(36.9)$ & $104(37.8)$ & $<0.0001$ \\
Age, years $($ mean $\pm \mathrm{SD})$ & $64.7(10.1)$ & $43.0(15.6)$ & $<0.0001$ \\
EQ5D-5L index value & $0.70(0.17)$ & $0.85(0.19)$ &
\end{tabular}

Data are expressed as $n(\%)$ unless otherwise specified

$S D$ standard deviation

a Low education level: lower technical and vocational training and lower general secondary education; medium education level: intermediate vocational training and advanced secondary education; high educational level: higher vocational education and university

Table 3 Results of mixed logit analysis for BWS case 2-patient and general population

\begin{tabular}{|c|c|c|c|c|c|}
\hline \multirow[t]{2}{*}{ Attribute } & \multirow[t]{2}{*}{ Levels } & \multicolumn{2}{|l|}{ Patient $(n=198)$} & \multicolumn{2}{|c|}{ General population $(n=276)$} \\
\hline & & Coefficient (SE) & $\begin{array}{l}\text { Attribute } \\
\text { impact (rank) }\end{array}$ & Coefficient (SE) & $\begin{array}{l}\text { Attribute } \\
\text { impact (rank) }\end{array}$ \\
\hline \multirow[t]{3}{*}{ Treatment modality } & Oral tablets & $3.51(0.25)^{\mathrm{a}}$ & & $2.72(0.16)^{\mathrm{a}}$ & \\
\hline & Pump & $-1.41(0.26)^{\mathrm{a}}$ & $1.0(1)$ & $1.23(0.18)^{\mathrm{a}}$ & $1.0(1)$ \\
\hline & Neurosurgery & $-4.93(0.62)^{\mathrm{a}}$ & & $-2.06(0.21)^{\mathrm{a}}$ & \\
\hline \multirow[t]{3}{*}{ Posture and balance problems } & Seldom to never & $3.47(0.19)^{\mathrm{a}}$ & & $3.63(0.13)^{\mathrm{a}}$ & \\
\hline & Sometimes & $1.52(0.20)^{\mathrm{a}}$ & $0.49(2)$ & $1.24(0.13)^{\mathrm{a}}$ & $0.84(3)$ \\
\hline & Often & $-0.68(0.23)^{\mathrm{a}}$ & & $-0.37(0.14)^{\mathrm{a}}$ & \\
\hline \multirow[t]{3}{*}{ Slowness of movement } & Seldom to never & $3.44(0.19)^{\mathrm{a}}$ & & $2.83(0.13)^{\mathrm{a}}$ & \\
\hline & Sometimes & $1.86(0.20)^{\mathrm{a}}$ & $0.46(3)$ & $1.66(0.13)^{\mathrm{a}}$ & $0.49(7)$ \\
\hline & Often & $-0.41(0.23)^{\mathrm{a}}$ & & $0.49(0.13)$ & \\
\hline \multirow[t]{3}{*}{ Tremor } & Seldom to never & $3.37(0.20)^{\mathrm{a}}$ & $0.42(4)$ & $3.45(0.13)^{\mathrm{a}}$ & $0.80(4)$ \\
\hline & Sometimes & $1.8(0.20)^{\mathrm{a}}$ & & $1.33(0.13)^{\mathrm{a}}$ & \\
\hline & Often & $-0.21(0.23)$ & & $-0.39(0.13)^{\mathrm{a}}$ & \\
\hline \multirow[t]{3}{*}{ Dyskinesia } & Seldom to never & $3.05(0.22)^{\mathrm{a}}$ & & $3.20(0.13)^{\mathrm{a}}$ & \\
\hline & Sometimes & $1.41(0.20)^{\mathrm{a}}$ & $0.36(5)$ & $1.46(0.13)^{\mathrm{a}}$ & $0.67(5)$ \\
\hline & Often & 0 (reference) & & 0 (reference) & \\
\hline \multirow[t]{3}{*}{ Dizziness } & Seldom to never & $2.75(0.20)^{\mathrm{a}}$ & & $3.51(0.13)^{\mathrm{a}}$ & \\
\hline & Sometimes & $1.53(0.21)^{\mathrm{a}}$ & $0.32(6)$ & $1.34(0.13)^{\mathrm{a}}$ & $0.90(2)$ \\
\hline & Often & $0.01(0.22)^{\mathrm{a}}$ & & $-0.78(0.13)$ & \\
\hline \multirow[t]{3}{*}{ Drowsiness } & Seldom to never & $2.83(0.2)^{\mathrm{a}}$ & 0.27 (7) & $2.91(0.13)^{\mathrm{a}}$ & $0.58(6)$ \\
\hline & Sometimes & $1.83(0.20)^{\mathrm{a}}$ & & $1.90(0.13)^{\mathrm{a}}$ & \\
\hline & Often & $0.57(0.22)^{\mathrm{a}}$ & & $0.12(0.13)$ & \\
\hline
\end{tabular}

$B W S$ best-worst scaling, $S E$ standard error

${ }^{a}$ Coefficients differing significantly from the value of the reference level $p<0.05$

shows that the relative order of treatment modalities is similar in both populations, with neurosurgery being perceived as most negative by both patients and the public (coordinates 0,0 ). Noteworthy is that patients valued the infusion of medication via a pump more negative, and the oral intake of medication slightly more positive, compared with the public view.

\subsection{Preference for Clinical Outcomes}

All health outcome attributes followed prior expectations, leading to decreasing scores as the level of impairment on each dimension increased. The two main differences in health outcomes between the patient and public populations were found in the perceived importance of the 


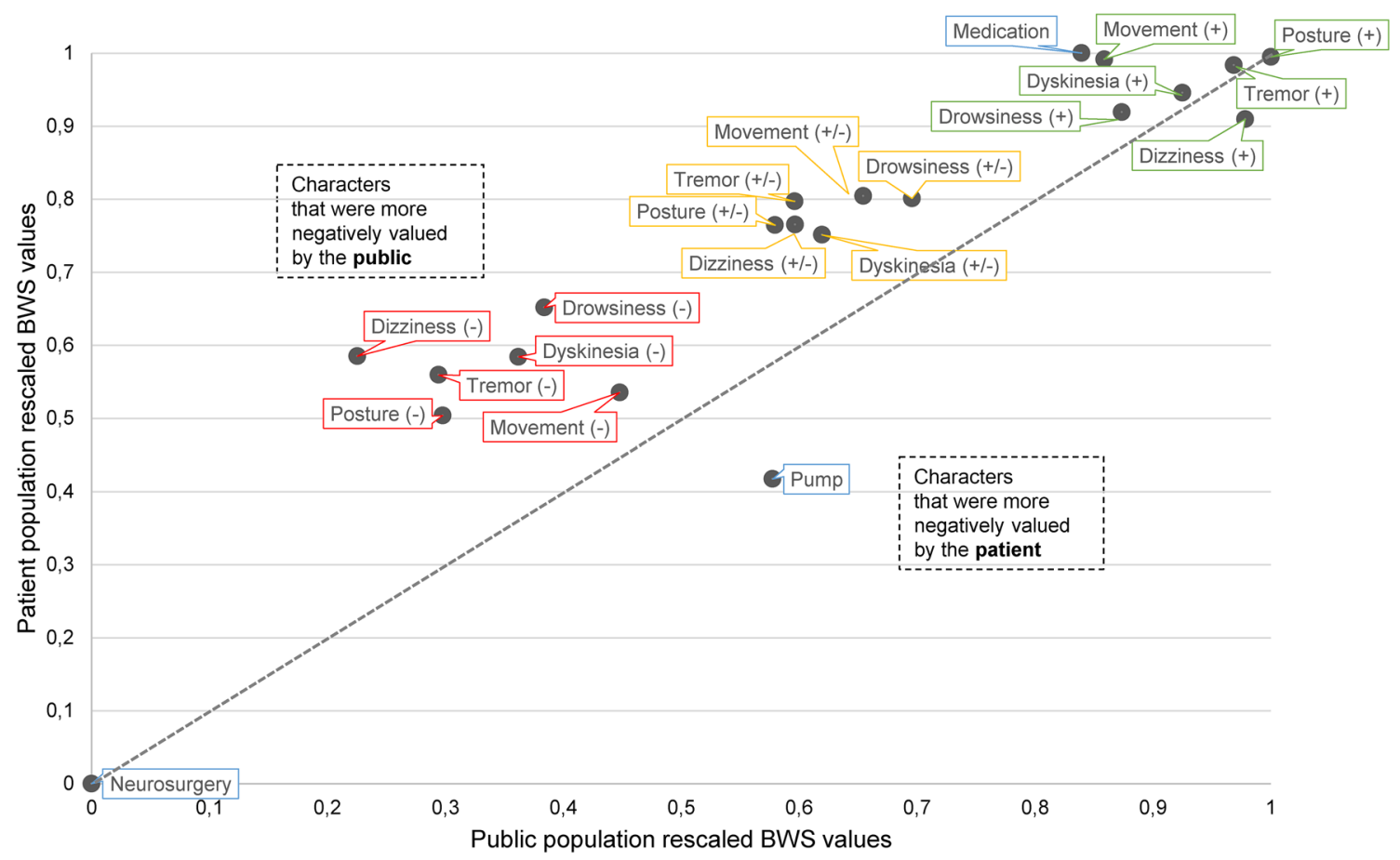

Fig. 2 Rescaled best-worst scaling, part-worth utilities for the patient and public populations. Blue represents treatment modality; red (-) represents 'often suffer from the symptom/side effect'; orange (+/-) represents 'sometimes suffer from symptom/side effect'; green (+) represents 'seldom to never suffer from symptom/side effect. Description: If the rescaled coefficient is on or close to the center line in this graph, patients and the public share the same

attributes 'slow movement' and 'dizziness'. Whereas for patients the effect of treatment on dizziness had less impact on their preference for treatment, the public found dizziness the second most important outcome to consider in choosing treatment. Conversely, slow movement, which was important to patients (third ranked), was the least important according to the public (seventh ranked). The differences in importance scores for the attributes 'drowsiness', 'tremor', 'posture and balance problems' and 'dyskinesia' were only minor.

\subsection{Subgroup Analysis of the Public Sample}

Fifty-five respondents (20\%) were familiar with PD because someone in their family, or a close friend, suffers from this condition. Notable here was that respondents who were familiar with PD assigned higher relative importance to tremors compared with respondents who were not familiar with PD (Fig. 3a). Figure 3b shows that respondents younger than the age of 30 years identified dizziness as the attribute that had the greatest impact on the perceived desirability of treatment, instead of treatment modality (which was favoured by the older age groups). Furthermore, older respondents assigned higher importance opinion on the specific attribute level (e.g. seldom to never suffer from drowsiness). The further the rescaled coefficient is from the center line, the more disagreement there exists between patients and public about this attribute-level (e.g. pump). The overall agreement between the two samples, based on Pearson's correlation coefficient, is $0.897(p<0.001)$. BWS best-worst scaling

to dyskinesia, while the younger respondents assigned higher importance to slow movement, dizziness and drowsiness. An interesting finding with regard to subgroups, based on quality of life, was that all respondents stated that treatment modality had the greatest impact on the perceived desirability of treatment (Fig. 3c). However, respondents with a quality-of-life score $<0.75$ assigned relatively higher importance to treatment modality compared with respondents with higher quality-of-life scores (lower attribute impacts for other attributes). Detailed tables with the results of the subgroup analysis can be found in "ESM Appendix C".

\section{Discussion}

The first objective of this study was to compare patient and public preferences for clinical outcomes (symptom reduction, side effects) of PD treatment. Most health outcomes were valued equally by patients and the public, except for reducing dizziness (more important to the public) and improving slow movement (more important to patients). In economic theory, it is assumed that importance of improving an outcome is directly linked to the perceived 


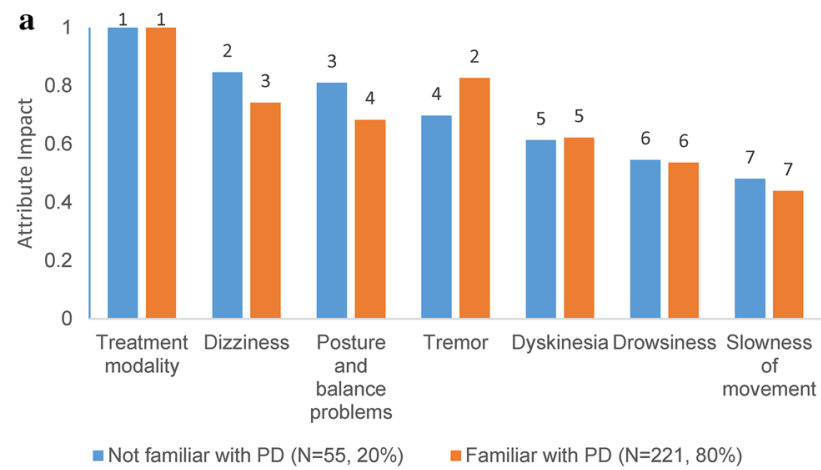

b

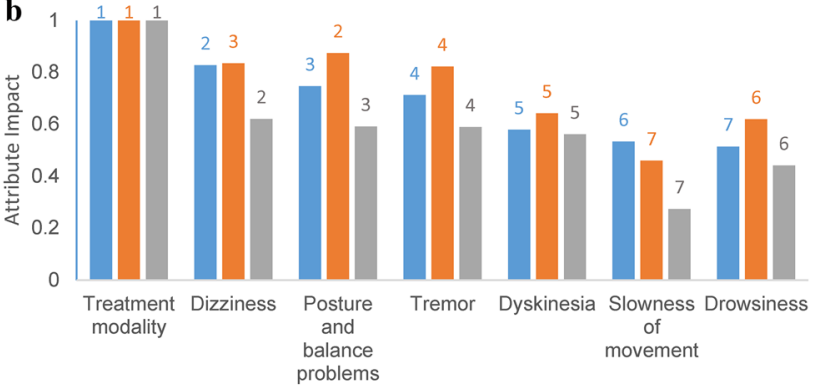

- $E Q 5 D=1(\mathrm{~N}=118,43 \%) \quad$ - $E Q 5 D=0.75-0.99(\mathrm{~N}=98,35 \%)$

$=E Q 5 D<0.75(N=60,22 \%)$

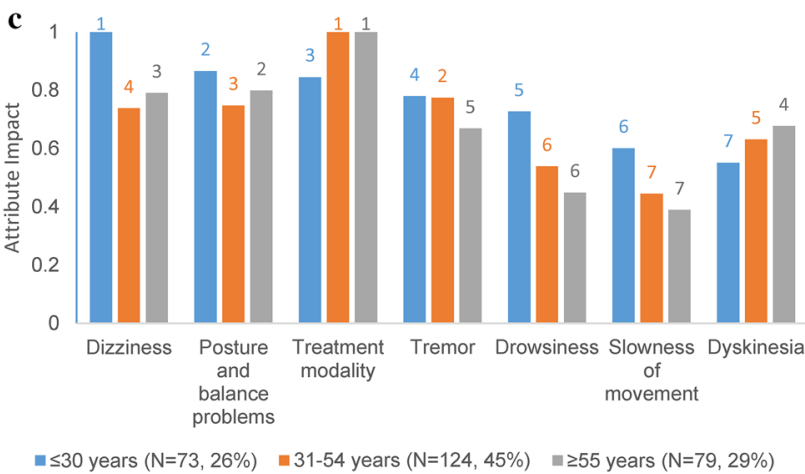

Fig. 3 Subgroup analysis of the preferences of public respondents. a Familiarity with PD; $\mathbf{b}$ quality of life of the respondents; $\mathbf{c}$ age of the respondents. $P D$ Parkinson's disease

severity of that outcome. Thus, it seems that relative to patients, the public underestimates the severity of slowness of movement and overestimates the severity of dizziness. A limitation of our experiment is that we did not quantify frequency of events. This might have influenced results, for instance because slowness of movement happens more frequently than dizziness in patients (and patients were aware of this), while dizziness is a more familiar experience to the public [28]. Conversely, slow movement, which was described as difficulty planning, initiating and executing movement, is an unfamiliar concept for non-PD patients, and thus its impact on life is difficult to determine for public respondents.

The second objective of this study was to compare the impact of treatment modality on treatment desirability from a patient and public perspective. This study clearly shows that the process of care is an important driver of preferences, in both patients and the general public; however, the patients assigned higher relative importance to treatment modality than the public population. In particular, the infusion of medication via a pump was valued more negatively by patients. In contrast to our expectations and hypothesis, the treatment modality can outweigh the benefits of PD treatments. Patients with PD might have adapted to their condition [29] and are not willing to undergo advanced treatments in order to receive health improvements.

Our last objective was to explore whether public preferences differ based on sociodemographic variables or the public respondents' familiarity with the disease. Subgroup analyses have shown that respondents who were familiar with PD assigned higher importance to the resting tremor than respondents who were not familiar with PD. A possible explanation is that tremor is one of the most prominent and common symptoms of PD and is therefore easily noticed. Furthermore, older respondents and respondents with a lower quality of life $(<0.75)$ have preferences that are more similar to the patients' preferences. They assign higher importance to the attribute 'treatment modality' compared with younger respondents and respondents with a higher quality of life. Just as with the patients, they are more concerned about process optimization and less focused on symptom relief and the prevention of side effects. Previous research has also shown that the expected burden of invasive treatments is an important reason for elderly patients to refuse medical interventions [30]. Older respondents and respondents with declining health may find it is easier to relate to PD patients, and, consequently, the preferences of these subgroups are more similar to the patients' preferences.

\subsection{Implications of Results}

Our study indicated differences between values of the patients and the general population. Although this study was not designed to recommend whether societal decision makers should add patients' values to their analysis, both public and patient preferences entail information that is potentially relevant for societal decision makers [31, 32]. For instance, knowing both perspectives can inform decision makers about the extent to which patients adapt to their condition by comparing patient and public utility estimates. Moreover, in this study, predicted uptake of invasive treatment is lower in patients compared with the public, which could indicate that desirability of a treatment is overestimated if its value is based on public preferences. This information could inform reimbursement and investment decision making [33]. Since patient and public 
preferences are not necessarily mutually exclusive, more initiatives are being employed to use patient preferences alongside public preferences in societal decision making $[20,34]$. The use of a stated preference survey in a representative sample of patients would be a valid method of doing so [35].

The results presented in this article (and previous research [19]) suggest that there are indications for preference heterogeneity in the patient and population data. The tendency for preferences to be determined by sociodemographics and experience is not new and has been found in other research [16-18]. For decision makers, the problem of preference heterogeneity can be added to the problem of whose values should count. More specifically, is preference heterogeneity relevant to measure, and, if so, how will it impact decisions?

\subsection{Strengths and Limitations}

To our knowledge, this was the first study to compare patient and public preferences of the relative importance of treatment aspects in PD. One difficulty encountered in pilot testing of the questionnaire was the cognitive difficulty of the choice task (especially for patients). In order to reduce cognitive burden, we used colour coding of the attribute levels, omitted quantitative frequency of symptoms and side effects, and did not randomize the order of attributes within or over the tasks. While these decisions may have influenced the elicitation of 'true' preferences, it is likely that its effect is similar in both populations. Furthermore, a limitation of our study was that public respondents received general information about $\mathrm{PD}$, while patients considered their own situation. This may have led to differences in the perception of (the benefit of) treatments and to variance both within and between patients and the public respondents.

In the analysis of our BWS data, we adopted the maxdiff model, in which it is assumed that respondents make a simultaneous choice of the pair of attribute levels that maximize the difference between them on a utility scale. However, respondents might have provided best-worst data in a particular order, which should be analyzed using a different model (sequential model of best-worst choices) [21]. Furthermore, we did not conduct latent class analysis to explore subgroups because several fit indices indicated a seven-class model which would be difficult to interpret given the aim of this study. Lastly, BWS experiments result in outcomes on a latent scale, therefore no direct comparisons can be made between two separate models without rescaling the data. In contrast to comparing two discrete choice models, no method is yet available to test for significant differences in utility estimations between two
BWS models [36]. This issue, as well as rescaling the BWS data on the health utility $(0-1)$ scale, are important areas for future research because the health utility scale is still favored in economic evaluations.

\section{Conclusions}

Our study has shown that patient and public judgments of the relative importance of treatment aspects in PD differ slightly. Patients assigned higher importance to treatment modality compared with the public population. Most health outcomes were valued equally by patients and the public, except for reducing dizziness (more important to the public) and improving slow movement (more important to patients). Public and patient preferences entail information that is potentially relevant for decision makers. Knowing the patients' preferences alongside the public preferences, for health outcomes and process of care, can inform decision makers about actual patient priorities and thus effective allocation of limited resources.

Author Contributions MW, JvT, CG-O, and MIJ conceived and designed the experiments; MW and JvT coordinated and conducted the experiments; and MW and CG-O conducted the statistical analysis and contributed to the interpretation of the data. All authors wrote the paper and provided critical input.

\section{Compliance with Ethical Standards}

Sources of funding This study was funded by ZonMw, the Netherlands Organization for Health Research and Development. Janine van Til received a grant to conduct this study (Grant Number 80-8250098-11234). Besides the initial review process prior to funding and amendments, ZonMw did not have any involvement in this study, and all research activities were independent of the funding source. The funders had no role in the study design, data collection and analysis, decision to publish, or in the preparation of the article.

Ethical standards According to the Medical Ethics Committee of the Medisch Spectrum Twente, our study did not require assessment according to the Medical Research (Human Subjects) Act. Following Dutch law, written informed consent was not required; however, by returning the completed questionnaire, patients agreed to the use of their anonymised data for research purposes in this study.

Conflicts of interest Marieke G. M. Weernink, Janine A. van Til, Catharina G. M. Groothuis-Oudshoorn, and Maarten J. IJzerman have indicated they have no conflicts of interest or financial interests to disclose.

Open Access This article is distributed under the terms of the Creative Commons Attribution-NonCommercial 4.0 International License (http://creativecommons.org/licenses/by-nc/4.0/), which permits any noncommercial use, distribution, and reproduction in any medium, provided you give appropriate credit to the original author(s) and the source, provide a link to the Creative Commons license, and indicate if changes were made. 


\section{References}

1. Lees AJ, Hardy J, Revesz T. Parkinson's disease. Lancet. 2009;373(9680):2055-66.

2. Martinez-Martin P, Kurtis MM. Health-related quality of life as an outcome variable in Parkinson's disease. Ther Adv Neurol Disord. 2012;5(2):105-17.

3. Schrag A, Jahanshahi M, Quinn N. What contributes to quality of life in patients with Parkinson's disease? J Neurol Neurosurg Psychiatry. 2000;69(3):308-12.

4. Clarke CE, Worth P, Grosset D, Stewart D. Systematic review of apomorphine infusion, levodopa infusion and deep brain stimulation in advanced Parkinson's disease. Parkinsonism Relat Disord. 2009;15(10):728-41.

5. Volkmann J, Albanese A, Antonini A, Chaudhuri KR, Clarke CE, de Bie RMA, et al. Selecting deep brain stimulation or infusion therapies in advanced Parkinson's disease: an evidence-based review. J Neurol. 2013;260(11):2701-14.

6. Banta D. The development of health technology assessment. Health Policy. 2003;63(2):121-32.

7. National Institute for Health and Clinical Excellence. Guide to the methods of health technology appraisal. London: National Institute for Health and Clinical Excellence; 2013.

8. Nederland Zorginstituut. Guideline for economic evaluation in healthcare. Diemen: Zorginstituut Nederland; 2016.

9. Stamuli E. Health outcomes in economic evaluation: who should value health? Br Med Bull. 2011;97:197-210.

10. Peeters Y, Stiggelbout AM. Health state valuations of patients and the general public analytically compared: a meta-analytical comparison of patient and population health state utilities. Value Health. 2010;13(2):306-9.

11. De Wit GA, Busschbach JJ, De Charro FT. Sensitivity and perspective in the valuation of health status: whose values count? Health Econ. 2000;9(2):109-26.

12. Coleman CI, Limone B, Sobieraj DM, Lee S, Roberts MS, Kaur $\mathrm{R}$, et al. Dosing frequency and medication adherence in chronic disease. J Manag Care Pharm. 2012;18(7):527-39.

13. Brennan VK, Dixon S. Incorporating process utility into quality adjusted life years: a systematic review of empirical studies. Pharmacoeconomics. 2013;31(8):677-91.

14. Wertheimer AI, Radican L, Jacobs MR. Assessing different perspectives on the value of a pharmaceutical innovation. South Med Rev. 2010;3(1):24-8.

15. Arbouw MEL, Movig KLL, Guchelaar HJ, Poels PJE, Van Vugt JPP, Neef C, et al. Discontinuation of ropinirole and pramipexole in patients with Parkinson's disease: clinical practice versus clinical trials. Eur J Clin Pharmacol. 2008;64(10):1021-6.

16. Ryan M, Ubach $C$. Testing for an experience endowment effect in health care. Appl Econ Lett. 2003;10(7):407-10.

17. Dolan P. The effect of experience of illness on health state valuations. J Clin Epidemiol. 1996;49(5):551-64.

18. Schaarschmidt ML, Umar N, Schmieder A, Terris DD, Goebeler $\mathrm{M}$, Goerdt S, et al. Patient preferences for psoriasis treatments: impact of treatment experience. J Eur Acad Dermatol Venereol. 2013;27(2):187-98.

19. Weernink MGM, van Til JA, van Vugt JPP, Movig KLL, Groothuis-Oudshoorn CGM, IJzerman MJ. Involving patients in weighting benefits and harms of treatment in Parkinson's disease. PloS One. 2016;11(8):e0160771.

20. Versteegh MM, Brouwer WBF. Patient and general public preferences for health states: a call to reconsider current guidelines. Soc Sci Med. 2016;165:66-74.

21. Louviere JJ, Flynn TN, Marley AAJ. Best-worst scaling theory, methods and applications. Cambridge: Cambridge University Press; 2015. p. 360.

22. Herdman M, Gudex C, Lloyd A, Janssen M, Kind P, Parkin D, et al. Development and preliminary testing of the new five-level version of EQ-5D (EQ-5D-5L). Qual Life Res. 2011;20(10):1727-36.

23. Johnson FR, Lancsar E, Marshall D, Kilambi V, Mühlbacher A, Regier DA, et al. Constructing experimental designs for discretechoice experiments: report of the ISPOR conjoint analysis experimental design good research practices task force. Value Health. 2013;16(1):3-13.

24. de Bekker-Grob EW, Donkers B, Jonker MF, Stolk EA. Sample size requirements for discrete-choice experiments in healthcare: a practical guide. Patient. 2015;8(5):373-84.

25. Hensher DA, Greene WH. The mixed logit model: the state of practice. Transportation. 2003;30(2):133-76.

26. Lancsar E, Louviere J, Flynn T. Several methods to investigate relative attribute impact in stated preference experiments. Soc Sci Med. 2007;64(8):1738-53.

27. Weernink MGM, Groothuis-Oudshoorn CGM, IJzerman MJ, van Til JA. Valuing treatments for Parkinson disease incorporating process utility: performance of best-worst scaling, time trade-off, and visual analogue scales. Value Health. 2016;19(2):226-32.

28. Ricci F, De Caterina R, Fedorowski A. Orthostatic hypotension: epidemiology, prognosis, and treatment. J Am Coll Cardiol. 2015;66(7):848-60.

29. Dakof GA, Mendelsohn GA. Patterns of adaptation to Parkinson's disease. Health Psychol. 1989;8(3):355-72.

30. Rothman MD, Van Ness PH, O'Leary JR, Fried TR. Refusal of medical and surgical interventions by older persons with advanced chronic disease. J Gen Intern Med. 2007;22(7):982-7.

31. Drummond M, Brixner D, Gold M, Kind P, McGuire A, Nord E. Toward a consensus on the QALY. Value Health. 2009;12(Suppl 1):S31-5.

32. Brazier J, Akehurst R, Brennan A, Dolan P, Claxton K, McCabe $\mathrm{C}$, et al. Should patients have a greater role in valuing health states? Appl Health Econ Health Policy. 2005;4(4):201-8.

33. Dowie J, Kjer Kaltoft M, Salkeld G, Cunich M. Towards generic online multicriteria decision support in patient-centred health care. Health Expect. 2015;18(5):689-702.

34. Abelson J, Wagner F, DeJean D, Boesveld S, Gauvin FP, Bean S, et al. Public and patient involvement in health technology assessment: a framework for action. Int $\mathbf{J}$ Technol Assess Health Care. 2016;32(4):256-64.

35. Danner M, Hummel JM, Volz F, Van Manen JG, Wiegard B, Dintsios CM, et al. Integrating patients' views into health technology assessment: Analytic hierarchy process (AHP) as a method to elicit patient preferences. Int J Technol Assess Health Care. 2011;27(4):369-75.

36. Swait J, Louviere J. The role of the scale parameter in the estimation and comparison of multinomial logit models. J Mark Res. 1993;30(3):305-14. 